\title{
Negative Area Compressibility in Silver Oxalate
}

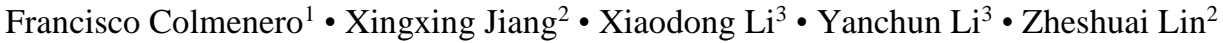

\begin{abstract}
The presence of extremely large negative linear compressibilities (NLC) in crystalline silver oxalate was discovered in a recent work by using first principles solid-state calculations. Although the minimum value of the NLC was found for a negative applied hydrostatic pressure, in this work the presence of NLC in this material for positive applied pressures is verified experimentally by means of high pressure X-ray diffraction experiments performed at room temperature in the Beijing Synchrotron Radiation Facility (BSRF). The results of this study demonstrate with certainty that the compressibility of silver oxalate along [010] crystallographic direction is negative for applied pressures in the range from 0.0 to $0.85 \mathrm{GPa}$. Since the measured compressibility decreases largely as the pressure decreases, large negative values of the compressibility are expected for negative applied pressures in agreement with the results found using first principles methods. Furthermore, the analysis of the variation of the lattice parameters of the crystal structure of silver oxalate under pressure in the principal axes reference system, revealed that this material also exhibits the largest negative area compressibility phenomenon found so far at zero pressure, the values of the compressibilities along two of the principal axes being -16.7 and $-20.0 \mathrm{TPa}^{-1}$. The negative compressibility phenomenon in silver oxalate can be rationalized in terms of a "chains of rotating parallelograms" structural model.
\end{abstract}

\section{Introduction}

In a recent paper [1], the presence of large negative linear compressibilities (NLC) [2-12] in crystalline silver oxalate, $\mathrm{Ag}_{2} \mathrm{C}_{2} \mathrm{O}_{4}$, was discovered by using high quality first principles solid-state calculations based in Density Functional Theory employing large plane wave basis sets and pseudopotential functions [13-14]. In fact, the absolute value of minimum compressibility found for silver oxalate, $-831.9 \pm 10 \mathrm{TPa}^{-1}$, is the largest found so far and the range of applied hydrostatic pressures for which this compound

\footnotetext{
* Francisco Colmenero

francisco.colmenero@iem.cfmac.csic.es

ORCID ID: https://orcid.org/0000-0003-3418-0735

* Xingxing Jiang

xxjiang@mail.ipc.ac.cn

ORCID ID: https://orcid.org/000-0001-6068-8773

* Zheshuai Lin

zslin@mail.ipc.ac.cn

ORCID ID: https://orcid.org/0000-0002-9829-9893

${ }^{1}$ Department of Molecular Physics, Instituto de Estructura de la Materia (IEM-CSIC), 28006 Madrid, Spain

2 Technical Institute of Physics and Chemistry, Chinese Academy of Sciences, Beijing 100190, China

${ }^{3}$ Beijing Synchrotron Radiation Facility, Institute of High Energy Physics, Chinese Academy of Sciences, Beijing 100049, China
}

exhibits NLC is also the widest found up to date [1]. The crystal structure of silver oxalate, its mechanical properties and the deformation of the crystal structure under pressure at the basis of the NLC phenomenon in this material were described in detail [1]. NLC effects were also found for oxalic acid [15-16] and other oxalate materials as ammonium oxalate monohydrate [17] and the anhydrous zinc and cadmium oxalates [18].

Although a wide range of important potential applications [2-3,10,12,19-21] for NLC materials have been found, the NLC phenomenon is quite infrequent [10] and the magnitude of the negative compressibility and the range of applied pressures for which these materials show NLC is frequently too small to be exploited in practical applications. The NLC effect found for silver oxalate [1] is very relevant not only due to its inherent significance. Since the differences in the magnitude of the minimum compressibility and NLC pressure range with respect to previous findings are very large, they indicate that the possibility of discovering even larger NLC effects is indeed significant. Silver oxalate is a compound which is used in photographic emulsions [22] and in a large series of technological applications [23-36] including the development of films for screen, electro-photographic and ink-jet printing [25-26]. In nanotechnology [23-34,26-28] it is widely used because the controlled thermal [38-31] or mechanical [32-35] decomposition of silver oxalate produces metallic nanoparticles with technologically desirable properties. In particular, silver nanoparticles have antimicrobial properties and are incorporated during the synthesis of compounds used as dental composites and in artificial bone materials [24,36]. Several easy high-yielding synthetic procedures [23,26,28,37-38] are known and, therefore, silver oxalate is a compound with large availability. The simplicity and availability of this compound suggest that the search of NLC materials should cover also common materials [12,15-16,18,39]. The presence of silver in several well-known NLC materials [40$43]$ is noteworthy.

While the results from high-quality first principles computations are generally in good agreement with experimental data, the predictions arising from this type of theoretical calculations may diverge from the expectations due to a large number of factors. One of the most important factors is the temperature because the first principles calculations correspond to zero temperature and the most interesting applications require that the NLC effect be observed at room temperature. Therefore, the predictions should always be corroborated by experimental measurements performed at interesting conditions. For this 
reason, an experimental high-pressure X-ray diffraction study of the negative linear compressibility effect in silver oxalate was carried out at the Beijing Synchrotron Radiation Facility (BSRF) [44].

\section{Materials and methods}

The experiments were performed with a crystalline powder sample of silver oxalate (CAS number 533-51-7, $99.02 \%$ purity) provided by Shangai Ichemical Co., Ltd. The compound was rigorously identified from X-ray diffraction experiments (see the next Section). The sample was used as received without further purification in the high pressure $\mathrm{X}$ ray diffraction experimental study.

The high-pressure X-ray diffraction experiments were performed at the $4 \mathrm{~W} 2$ beam line of Beijing Synchrotron Radiation Facility [44]. The X-ray beam at the wavelength $0.6199 \AA$ was focused into a $36 \times 12 \mu \mathrm{m}^{2}$ spot using Kirkpatrick-Baez mirrors. The hydrostatic pressure was exerted by the systematic diamond anvil cells (DAC) [45] with the culet diameter of $400 \mu \mathrm{m}$. The samples in well grinded powder were placed in a hole of about $120 \mu \mathrm{m}$ diameter in a pre-indented stainless steel gasket with the thickness of $40 \mu \mathrm{m}$. Silicon oil was adopted to act as the pressure-transmitting medium and ruby chips were placed for pressure calibration by measuring the fluorescence shift as a function of pressure [46]. The diffraction patterns were recorded by a Pilatus image plate and integrated with the FIT2D software package.

\section{Results and discussion}

The X-ray diffraction powder patterns of silver oxalate obtained at four different applied hydrostatic pressures $(0.0$, $0.134,0.478$ and $0.848 \mathrm{GPa}$ ) are shown in Fig. 1. The four patterns are provided as Supplementary Material. The reference X-ray powder pattern of silver oxalate was determined in our previous work [1] from the experimental unit cell reported by Naumov et al. [47] in 1995. The measured X-ray diffraction powder pattern at zero pressure is compared with the reference pattern in Fig. S.1 of the Supplementary Material. The precise values of the positions of the most important reflections in the powder pattern at 0.0 $\mathrm{GPa}$ and the corresponding data from the reference pattern are given in Table S.1. Both sets of data are in excellent agreement, the largest deviations being about $0.02 \AA$. These results confirm the good quality of the sample used in the present study. However, the presence of two extra peaks in the measured $\mathrm{X}$-ray diffraction pattern at $15.12^{\circ}$ and $17.47^{\circ}$ must be noticed. These two peaks are due to the presence of an impurity of pure metallic silver without impact on the general crystallinity of the sample as shown in Fig. S.2 of the Supplementary Material.

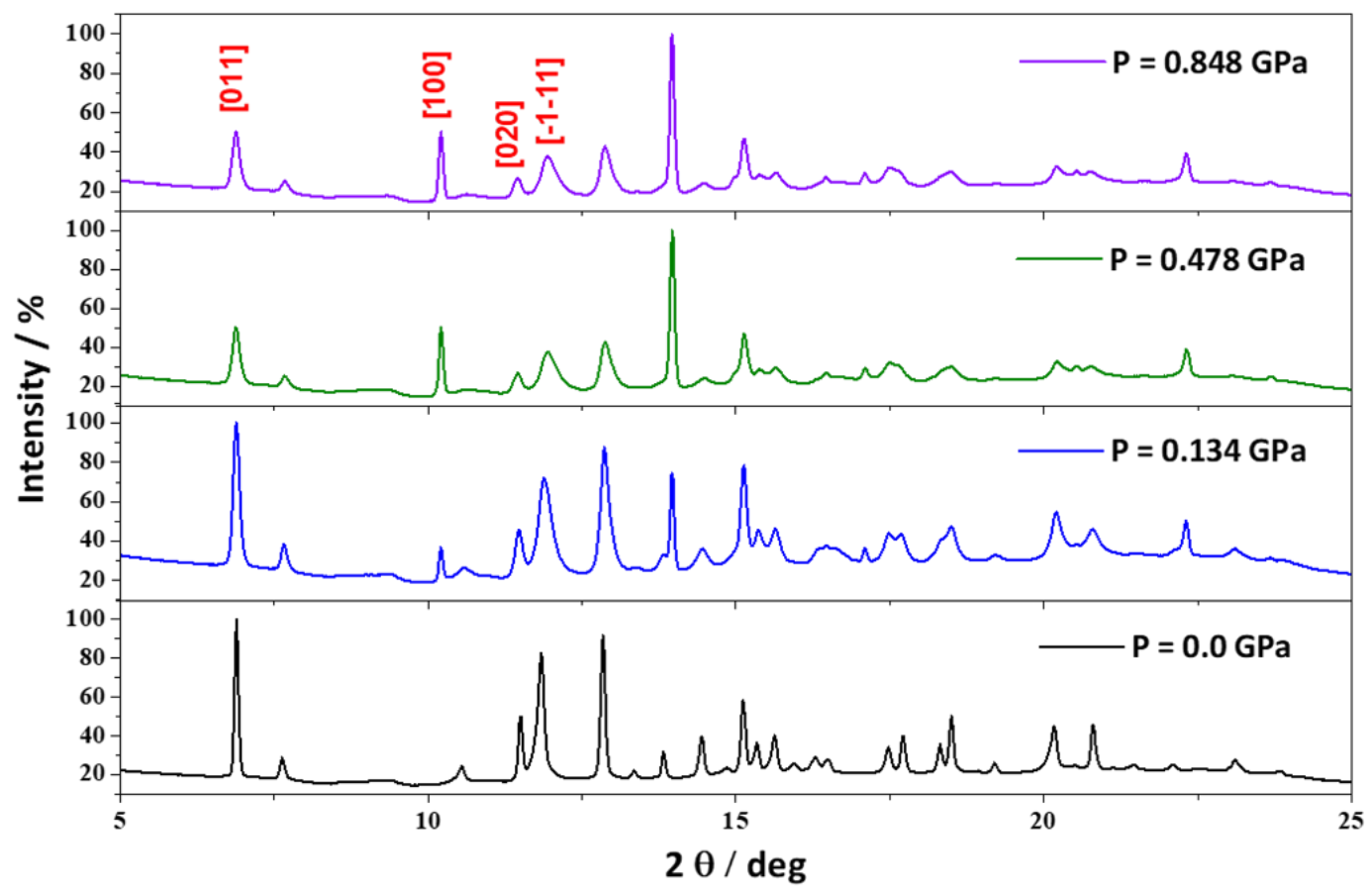

Fig. 1. X-ray powder patterns of silver oxalate under pressure. The position of the [011], [100], [020] and [-1-11] reflections is indicated.

Four well-defined reflections in the X-ray powder pattern were selected to extract the lattice parameters of the crystal structure of silver oxalate. These reflections are [-1-11], [011], [020] and [100]. The expressions used to derive the lattice parameters from the positions of these reflections are given in Appendix A. The values of the lattice parameters obtained at the four different pressures are reported in Table 1. Again, the lattice parameters obtained at zero pressure are in very good agreement with the reference data from Naumov et al. [47] (given also in Table 1), the values of the 
$b$ lattice parameter differing by less than $0.1 \%$. As can be seen in Table 1, the lattice parameters obtained using firstprinciples calculations [1] are also in good agreement with the experimental results, the computed unit-cell volume differing from the experimental one by $1.1 \%$, From the computed crystal structure the mechanical properties of silver oxalate were determined theoretically [1]. A short survey of the results obtained, including the bulk modulus and its pressure derivatives as well as the computed elasticity matrix are given in Table S.2 of the Supplementary Material.

Table 1. Lattice parameters of silver oxalate under pressure. The parameters at zero pressure are compared with the parameters from the reference pattern from Naumov et al. [47] and those obtained in the first principles calculations [1].

\begin{tabular}{|c|c|c|c|c|c|c|}
\hline \multirow{2}{*}{ Parameter } & \multicolumn{3}{|c|}{$P=0.0 \mathrm{GPa}$} & \multirow{2}{*}{$\mathrm{P}=0.134 \mathrm{GPa}$} & \multirow{2}{*}{$P=0.474 \mathrm{GPa}$} & \multirow{2}{*}{$P=0.848 \mathrm{GPa}$} \\
\hline & Naumov et al. [47] & DFT [1] & Present work & & & \\
\hline$a(\AA)$ & $3.4603(5)$ & 3.5713 & 3.4765 & 3.4608 & 3.4381 & 3.4138 \\
\hline$b(\AA)$ & $6.1972(9)$ & 6.0319 & 6.1932 & 6.2058 & 6.2184 & 6.2317 \\
\hline$c(\AA)$ & $9.548(2)$ & 9.4867 & 9.5796 & 9.5492 & 9.5323 & 9.3933 \\
\hline$\beta$ (deg) & $103.47(10)$ & 105.5823 & 103.6911 & 103.3643 & 102.9055 & 100.9885 \\
\hline Vol. $\left(\AA^{3}\right)$ & $199.12(6)$ & 196.8535 & 200.3926 & 199.5341 & 198.6475 & 196.1681 \\
\hline
\end{tabular}

As can be observed in Table 1, the $b$ lattice parameter of silver oxalate increases invariably from 0.0 to $0.848 \mathrm{GPa}$. These results confirm with certainty the negative linear compressibility phenomenon in silver oxalate, in agreement with the results of the first principles calculations [1] at zero temperature. The phenomenon persists at room temperature. In order to determine the compressibility of silver oxalate at zero pressure from the structural data obtained in the high pressure experiments, the approach proposed by Cliffe and Goodwin [48] in 2012 was used. Thus, the PASCal online tool [49] was employed. The values obtained for the linear compressibilities in the principal axes reference system are given in Table 2.

Table 2. Linear compressibilities of silver oxalate in the principal axes reference system.

\begin{tabular}{cc}
\hline Principal axis $\left(\boldsymbol{u}_{\boldsymbol{i}}\right)$ & $\boldsymbol{k}_{\boldsymbol{i}}\left(\mathbf{T P a}^{-\mathbf{1}}\right)$ \\
\hline$u_{1}=[0.9791,0.0,-0.2033]$ & 61.36 \\
$u_{2}=[0.0,1.0,0.0]$ & -16.73 \\
$u_{3}=[0.8910,0.0,0.4540]$ & -19.98 \\
\hline
\end{tabular}

To test the values obtained using PASCal software, a fit of the values of the $b$ lattice parameter (as a function of the applied pressure) to a Birch-Murnahan [50] equation of state (BM-EOS) was performed using Angel's EOSFIT 5.2 software [51-52]. From the fitted value of the bulk modulus, the compressibility along $b$ direction, $k_{b}=-1 / b \cdot(\partial b /$ $\partial P)_{P}$, was obtained. Since the [010] crystallographic direction coincides with the second principal axis, the value obtained for $k_{b}$ agreed very well with the value of $k_{2}$ given in Table 2. The final value for the compressibility along [010] direction, including the standard deviation obtained with EOSFIT code, is $k_{2}=-16.7 \pm 0.2 \mathrm{TPa}^{-1}$.

From the results given in Table 2, it follows that the linear compressibilities of silver oxalate along two directions of the principal axes are negative. Therefore, silver oxalate displays the negative area compressibility phenomenon $[10,16,43,53-59]$ at zero pressure. The compressibility along the first principal axis is positive and very large to compensate for the negative values of the compressibilities along the second and third axes. The linear compressibilities obtained for silver oxalate are compared in Table S.3 of the Supplementary material with a selection of values of the compressibilities for the most important NLC materials at zero pressure known so far. The absolute value of the negative compressibilities, $k_{2}$ or $k_{3}$, is larger than those of most materials, except $\mathrm{CsH}_{2} \mathrm{PO}_{4}$ [60], $\mathrm{Ag}_{3}\left[\mathrm{Co}(\mathrm{CN})_{6}\right]$ [4142], $\mathrm{Zn}\left[\mathrm{Au}(\mathrm{CN})_{2}\right]_{2}$ [61], MIL-53 [62] and $\mathrm{InH}(\mathrm{BDC})_{2}$ [63]. However, the absolute value of the compressibility of silver oxalate along $b$ direction obtained from first principles calculations at $\mathrm{P}=-0.16 \mathrm{GPa}$ is much larger than the compressibilities in these materials.

Since for silver oxalate the compressibilities along two directions are negative, the comparison with other systems exhibiting NAC is more appropriate. The value of the largest compressibility in three different NAC materials, $\mathrm{NaV}_{2} \mathrm{O}_{5}$ [53-55], $\mathrm{TlGaSe}_{2}$ [56] and $\mathrm{Ag}\left[\mathrm{C}(\mathrm{CN})_{3}\right]$ [43] are given in the last rows of Table S.3. As can be seen, the absolute values of the compressibilities in silver oxalate are much larger than those of these materials.

In order to compare the values of the compressibilities obtained in the first principles calculations with the experimental values at non-zero pressures, the values of the compressibility, $k_{b}=-1 / b \cdot(\partial b / \partial P)_{P}$, were evaluated at selected pressures between 0.10 and $0.20 \mathrm{GPa}$ from the BMEOS. The results are given in Table 3. For example, at 0.13 $\mathrm{GPa}$, the compressibility is $k_{b}=-11.7 \mathrm{TPa}^{-1}$, which may be compared with the theoretical value of $-21.3 \mathrm{TPa}^{-1}$. The agreement is reasonable in view of the fact that the theoretical result corresponds to zero temperature and the experimental one to room temperature and taking into account the approximate nature of the first principles calculations. The variation of the measured $b$ lattice parameter and associated compressibility at small external pressures is displayed in Fig. 2. The absolute value of the compressibility increases drastically as the pressure decreases. Therefore, large negative compressibilities at negative pressures are expected in agreement with the results obtained in the first principles work [1]. 
Table 3. Comparison of the compressibilities of silver oxalate for a selected set of pressures in the range from 0.1 to $0.2 \mathrm{GPa}$ with the values obtained from first principles calculations [1].

\begin{tabular}{ccc}
\hline $\begin{array}{c}\text { Pressure } \\
(\mathbf{G P a})\end{array}$ & $\begin{array}{c}\text { Exp. } \\
\boldsymbol{k}_{\boldsymbol{b}}\left(\mathbf{T P a}^{-1}\right)\end{array}$ & $\begin{array}{c}\mathbf{D F T} \\
\boldsymbol{k}_{\boldsymbol{b}}\left(\mathbf{T P a}^{-\mathbf{1}}\right)\end{array}$ \\
\hline 0.11 & -12.7 & -27.5 \\
0.13 & -11.7 & -21.3 \\
0.15 & -10.7 & -18.5 \\
0.17 & -9.8 & -18.0 \\
0.19 & -9.0 & -19.0 \\
\hline
\end{tabular}

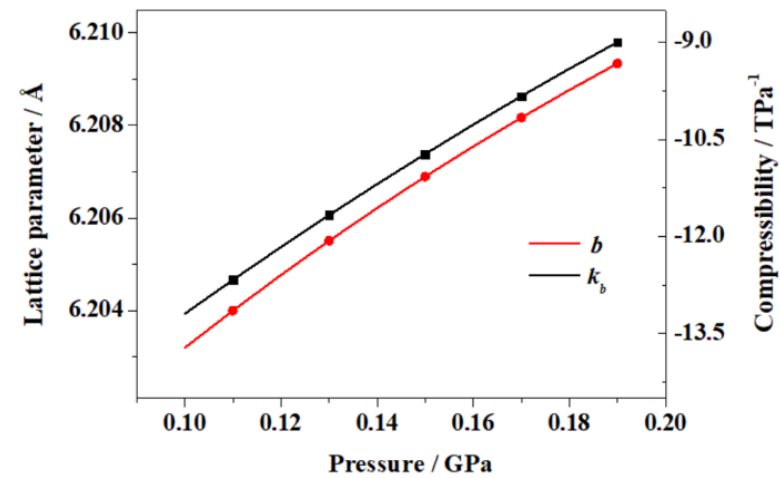

Fig. 3. Experimental $b$ lattice parameter and compressibilities of silver oxalate for small external pressures.

\section{NLC mechanism in silver oxalate}

The structural mechanism leading to the presence of NLC in silver oxalate, studied in the earlier paper [1], was reinvestigated in this work. Figs. 3.A and Fig. 3.B provide views of the crystal structure of silver oxalate under the effect of two different increasing isotropic pressures -0.394 and $-0.127 \mathrm{GPa})$ parallel to $(y z)$ and $(x z)$ planes. Under compression, the $b$ lattice parameter increases strongly and the other two parameters decrease to compensate the increase of $b$ so that the total unit-cell volume decreases. An inspection of the views of the crystal structure of silver oxalate, provided in Fig . 3, shows the presence of chains of parallelograms expanding along [010] and [100] direction connected by the oxalate groups. The parallelograms do not share an edge but a vertex (occupied by a silver atom) and are rotated relative to each other. These chains, isolated of the structure, are displayed in Fig. 3. As is well-known [6467], many materials contain a connected-squares motif in one of their cross-sections which is responsible of their anomalous mechanical behavior. In the present case, the behavior under compression of the chains of rotating parallelograms forces the increase the dimension of the full structure along [010] and the diminution of its dimension along [100]. Since the chains are not linked directly, the structural model differs from the rotating squares model [63] or other models based on rotating polygons as rectangles [65,68], rhombi [69] and parallelograms [70-71]. An analytical study of the properties of this model should be performed in order to analyze the variations of its mechanical properties with respect to the variations in the structural parameters of the model such as the length of the edges of the parallelograms, their internal angle and the distances between the chains.

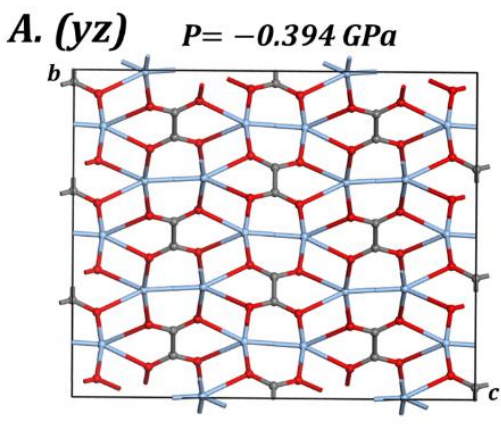

B. (XZ) $P=-0.394 \mathrm{GPa}$

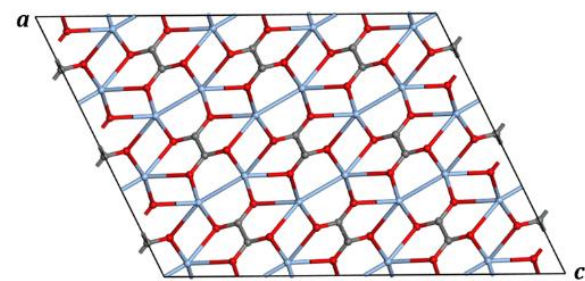

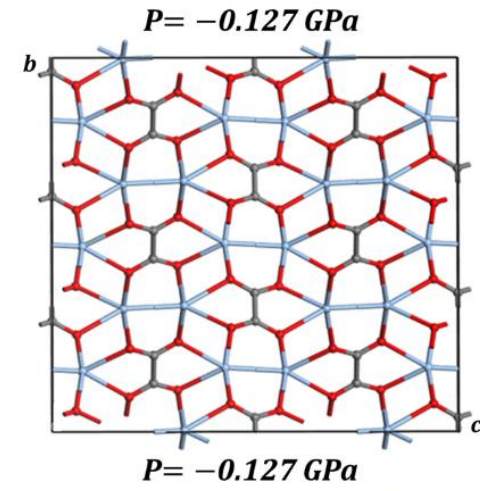

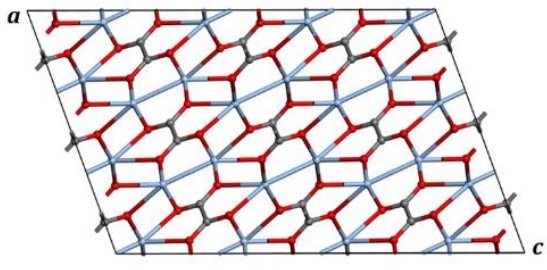

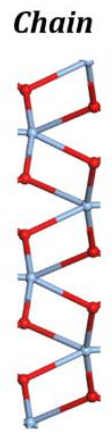

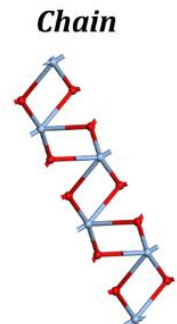

Fig. 3. Views of $3 \times 3 \times 2$ supercells of the computed crystal structures of silver oxalate [1] under isotropic pressures $\mathrm{P}=-0.394 \mathrm{GPa}$ and $\mathrm{P}=-0.127 \mathrm{GPa}$ : (A) $y z$ plane; (B) $x z$ plane; (C) Isolated chains of rotating parallelograms. Color code: Ag - Clear blue; $\mathrm{C}-\mathrm{Gray}$; $\mathrm{O}$ - Red. 


\section{Conclusions}

In this work, the X-ray diffraction patterns of a powdered crystalline sample of silver oxalate under pressure were measured at room temperature in the Beijing Synchrotron Radiation Facility. From the X-ray powder patterns, the lattice parameters of the crystal structure of this material under different isotropic pressures were extracted. The analysis of these parameters revealed the extremely anomalous mechanical behavior of this material. The unit cell of silver oxalate increases along the directions of two of its principal axes under compression and, therefore, silver oxalate exhibits the highly infrequent negative area compressibility phenomenon. The compressibilities along these two directions are not only negative but also very large in absolute value. Silver oxalate presents the more extreme NAC effect found so far.

These results constitute an experimental verification of the negative linear compressibility effect predicted earlier for silver oxalate using first principles solid state methods [1]. The prediction was made for silver oxalate at zero temperature, but the NLC effect in this material persists at room temperature. Therefore, the first principles methodology allows to perform accurate predictions of the mechanical properties of solid materials. At the same time, the accuracy of these methods for mechanical property prediction probes their validity for the interpretation and visualization of the deformation of the structures under pressure at the microscopic scale, being a powerful tool for unveiling the mechanisms leading to the presence of anomalous mechanical phenomena in solid materials. An inspection of the computed crystal structures of silver oxalate under pressure, reveals that the negative compressibility mechanism in silver oxalate can be rationalized in terms of a "chains of rotating parallelograms" structural model.

\section{Appendix A. Determination of the lattice parameters of silver oxalate}

For crystalline systems with monoclinic space symmetry, the position of the reflections with indices $[h 00],[0 k 0]$, $[0 k l]$ and $[h k l]$ are given by [72]:

$$
\begin{gathered}
d[h 00]=\frac{a}{h} \sin \beta \\
d[0 k 0]=\frac{b}{k} \\
d[0 k l]=\left(\frac{k^{2}}{b^{2}}+\frac{l^{2}}{(c \sin \beta)^{2}}\right)^{-\frac{1}{2}} \\
d[h k l]=\left(\frac{h^{2}}{(a \sin \beta)^{2}}+\frac{k^{2}}{b^{2}}+\frac{l^{2}}{(c \sin \beta)^{2}}-\frac{2 h l \cos \beta}{(a \sin \beta)(a \sin \beta)}\right)^{-\frac{1}{2}}
\end{gathered}
$$

From the positions of the [020], [100], [011] and [-1-11] reflections, one can determine all the lattice parameters. From (A.2), we can obtain $b$ :

$$
b=2 \cdot d[020]
$$

and from (A.1), we can determine $a \sin \beta$ using:

$$
\text { as } \equiv a \sin \beta=d[100]
$$

Now from (A.3), since $b$ is known, one can obtain $c \sin \beta$ :

$$
c s \equiv c \sin \beta=\left(\frac{1}{d[011]^{2}}-\frac{1}{b^{2}}\right)^{-\frac{1}{2}}
$$

Finally, from (A.4) we obtain $\cos \beta$ (and $\beta$ itself),

$$
\cos \beta=\frac{a s \cdot c s}{2}\left(\frac{1}{d[-1-11]^{2}}-\frac{1}{b^{2}}-\frac{1}{a s^{2}}-\frac{1}{c s^{2}}\right)
$$

The determination of $a$ and $c$ from $a s, b s$ and $\beta$ is obvious.

\section{Acknowledgements}

This work was supported by the National Scientific Foundations of China (Grants 51702330, 51872297 and 11974360). FC wants to thank the help of Dr. A. M. Fernández for the help with the analysis of the X-ray diffraction pattern of silver oxalate.

\section{Compliance with Ethical Standards}

Conflict of Interest: The authors declare that they have no conflict of interest.

\section{References}

1. Colmenero F (2019) Silver Oxalate: Mechanical Properties and Extreme Negative Mechanical Phenomena. Adv Theor Simul 2: 1900040

2. Baughman RH, Stafström S, Cui C, Dantas SO (1998) Materials with Negative Compressibilities in One or More Dimensions. Science 279: 1522-1524

3. Kornblatt JA, Sirota EB, King HE, Baughman RH, Cui C (1998) Materials with Negative Compressibilities. Science 281: 143-143

4. Lakes RS, Wojciechowski KW (2008) Negative Compressibility, Negative Poisson's Ratio and Stability. Phys Stat Sol (b) 245: 545551

5. Weng CN, Wang KT, Chen, T (2008) Design of Microstructures and Structures with Negative Linear Compressibility in Certain Directions. Adv Mater Res 33-37: 807-814

6. Grima JN, Attard D, Caruana-Gauci R, Gatt R (2011) Negative linear compressibility of hexagonal honeycombs and related systems. Scr Mater 65: 565-568

7. Grima JN, Caruana-Gauci R, Attard D, Gatt R (2012) Threedimensional cellular structures with negative Poisson's ratio and negative compressibility properties. Proc Roy Soc A 468: 31213138

8. Grima JN, Caruana-Gauci R (2012) Mechanical metamaterials: Materials that push back. Nat Mater 11: 565-566

9. Barnes DL, Miller W, Evans KE, Marmier ASH (2012) Modelling negative linear compressibility in tetragonal beam structures. Mech Mater 46: 123-128

10. Cairns AB, Goodwin L (2015) Negative Linear Compressibility. Phys Chem Chem Phys 17: 20449-20465 
11. Binns J, Kamenev KV, Marriott KER, McIntyre GJ, Moggach SA, Murrie M, Parsons S (2016) A non-topological mechanism for negative linear compressibility. Chem Commun 52: 7486-7489

12. Colmenero F (2019) Anomalous mechanical behavior of the deltic, squaric and croconic cyclic oxocarbon acids. Mater Res Express 6: 045610

13. Payne MC, Teter MP, Ailan DC, Arias A, Joannopoulos JD (1992) Iterative minimization techniques for ab initio total-energy calculations: molecular dynamics and conjugate gradients. Rev Mod Phys 64: 1045-1097

14. Clark SJ, Segall MD, Pickard CJ, Hasnip PJ, Probert MIJ, Refson K, Payne MC (2005) First Principles Methods Using CASTEP. Z Kristallogr 220: 567-570

15. Colmenero F (2019) Mechanical Properties of Anhydrous Oxalic Acid and Oxalic Acid Dihydrate. Phys Chem Chem Phys 21: 2673-2690

16. Colmenero F (2019) Negative Area compressibility in oxalic acid dihydrate. Mater Lett 245: 25-28

17. Qiao Y, Wang K, Yuan H, Yang K, Zou B (2015) Negative Linear Compressibility in Organic Mineral Ammonium Oxalate Monohydrate with Hydrogen Bonding Wine-Rack Motifs. J Phys Chem Lett 6: 2755-2760

18. Colmenero F, Timón V (2020) Extreme negative mechanical phenomena in the zinc and cadmium anhydrous metal oxalates and lead oxalate dihydrate. J Mater Sci 55: 218-236

19. Evans KE, Alderson A (2000) Auxetic Materials: Functional Materials and Structures from Lateral Thinking! Adv Mater 12: 617-628

20. Spinks GM, Wallace GG, Fifield LS, Dalton LR, Mazzoldi A, De Rossi D, Khayrullin I, Baughman RH (2002) Pneumatic Carbon Nanotube Actuators. Adv Mater 14: 1728-1732

21. Aliev AE, Oh J, Kozlov ME, Kuznetsov AA, Fang S, Fonseca AF, Ovalle R, Lima MD, Haque MH, Gartstein YN, Zhang M, Zakhidov AA, Baughman RH (2009) Giant-Stroke, Superelastic Carbon Nanotube Aerogel Muscles. Science 323: 1575-1578

22. Sheppard SE, Wanselow W (1934) Photothermographic composition. United States Patents US1976302A

23. Navaladian S, Viswanathan B, Viswanath RP, Varadarajan TK (2007) Thermal decomposition as route for silver nanoparticles. Nanoscale Res Lett 2: 44-48

24. Zhiteneva DA, Sverdlova NI, Mikhalchan AA, Astashkina OV (2011) Some features of production of silver-containing carbon materials. Fibre Chem 42: 313-317

25. Lin HC, Lin P, Lu CA, Wang SF (2009) Effects of silver oxalate additions on the physical characteristics of low-temperaturecuring MOD silver paste for thick-film applications. Microelectron Eng 86: 2316-2319

26. Dong Y, Li X, Liu S, Zhu Q, Li JG, Sun X (2015) Facile synthesis of high silver content MOD ink by using silver oxalate precursor for inkjet printing applications. Thin Solid Films 589: 381-387

27. Zhang S, Tang Y, Vlahovic B (2016) A Review on preparation and applications of silver-containing nanofibers. Nanoscale Res Lett 11: 80-87

28. Sarada K, Vijisha KR, Muraleedharan K (2016) Exploration of the thermal decomposition of oxalates of copper and silver by experimental and computational methods. J Anal Appl Pyrol 120: 207-214

29. MacDonald JY, Hinshelwood CN (1925) The formation and growth of silver nuclei in the decomposition of silver oxalate. J Chem Soc Trans 127: 2764-2771

30. Dollimore D (1987) The thermal decomposition of oxalates. A review. Thermochim Acta 117: 331-363
31. Boldyrev VV (2002) Thermal decomposition of silver oxalate. Thermochim Acta 388: 63-90

32. Delogu F (2012) Estimation of the mass of powder trapped at collision during ball milling from the kinetics of the mechanically activated decomposition of Ag oxalate. Mater Chem Phys 137: 297-302

33. Delogu F (2014) Mechanochemical decomposition of Ag and Ni oxalates. Mater Chem Phys 147: 629-635

34. Ligios GA, Bertetto M, Delogu F (2013) A systematic investigation of the mechanochemical decomposition of $\mathrm{Ag}$ oxalate in rod drop experiments. J Alloys Compd 554: 426-431

35. Delogu F (2014) Ag-Ni Janus nanoparticles by mechanochemical decomposition of Ag and Ni oxalates. Acta Mater 66: 388-395

36. Stencel R, Kasperski J, Pakieła W, Mertas A, Bobela F, Barszczewska-Rybarek I, Chladek G (2018) Properties of experimental dental composites containing antibacterial silverreleasing filler. Materials 11: 1031-1057

37. Donia AM (1997) Synthesis, identification and thermal analysis of coprecipitates of silver-(cobalt, nickel, copper and zinc) oxalate. Polyhedron 16: 3013-3031

38. Pourmortazavi SM, Taghdiri M, Makari V, Nasrabadi MR (2015) Procedure optimization for green synthesis of silver nanoparticles by aqueous extract of Eucalyptus oleosa. Spectrochim Acta A (2015) 136: 1249-1254

39. Colmenero F (2020) Organic acids under pressure: elastic properties, negative mechanical phenomena and pressure induced phase transitions in the lactic, maleic, succinic and citric acids. Mater Adv 1, 1399-1426

40. Goodwin AL, Keen DA, Tucker MG (2008) Large negative linear compressibility of $\mathrm{Ag}_{3}\left[\mathrm{Co}(\mathrm{CN})_{6}\right]$. Proc Natl Acad Sci USA 105: 18708-18713

41. Goodwin AL, Calleja M, Conterio MJ, Dove MT, Evans JSO, Keen DA, Peters L, Tucker MG (2008) Colossal Positive and Negative Thermal Expansion in the Framework Material $\mathrm{Ag}_{3}\left[\mathrm{Co}(\mathrm{CN})_{6}\right]$. Science 319: 794-797

42. Cairns AB, Thompson AL, Tucker MG, Haines J, Goodwin AL (2012) Rational Design of Materials with Extreme Negative Compressibility: Selective Soft-Mode Frustration in $\mathrm{KMn}\left[\mathrm{Ag}(\mathrm{CN})_{2}\right]_{3}$. J Am Chem Soc 134: 4454-4456

43. Hodgson SA, Adamson L, Hunt SJ, Cliffe MJ, Cairns AB, Thompson AL, Tucker MG, Funnella NP, Goodwin AL (2014) Negative Area Compressibility in Silver(I) Tricyano-methanide. Chem Commun 50: 5264-5266

44. Liu J (2016) High pressure x-ray diffraction techniques with synchrotron radiation. Chin Phys B 25: 076106

45. Eremets MI (1996) Diamond Anvil Cell. In: Eremets MI (1996) High Pressure Experimental Methods. Oxford University Press, Oxford. Ch 3

46. Mao HK, Xu, J, Bell PM (1986) Calibration of the ruby pressure gauge to 800 kbar under quasi-hydrostatic conditions. J Geophys Res: Solid Earth 91: 4673-4676

47. Naumov DY, Virovets AV, Podberezkaya NY, Boldyreva EV (1995) Silver Oxalate, $2 \mathrm{Ag}^{+} \cdot \mathrm{C}_{2} \mathrm{O}_{4}^{2-}$. Acta Crystallogr C 51: 6062

48. Cliffe MJ, Goodwin AL (2012) PASCal: a principal axis strain calculator for thermal expansion and compressibility determination. J Appl Cryst 45: 1321-1329

49. PASCal, A web tool for Principal Axis Strain Calculations, http://pascal.chem.ox.ac.uk/ (accessed 15 May 2019)

50. Birch F (1947) Finite Elastic Strain of Cubic Crystals. Phys Rev 71: 809-824 
51. Angel RJ (2000) Equations of State. Rev Mineral Geochem 41: $35-60$

52. EOSFIT 5.2, http://programming.ccp14.ac.uk/ccp/web-mirrors/ ross-angel/crystal/ software.html (accessed 15 May 2019)

53. Loa I, Syassen K, Kremer R, Schwarz U, Hanfland M (1999) Structural properties of $\mathrm{NaV}_{2} \mathrm{O}_{5}$ under high pressure. Phys Rev B 60: R6945-R6948

54. Loa I, Syassen K, Kremer R (1999) Vibrational Properties of $\mathrm{NaV}_{2} \mathrm{O}_{5}$ under High Pressure Studied by Raman Spectroscopy. Solid State Commun 112: 681-685

55. Ohwada K, Nakao H, Fujii Y, Isobe M, Ueda Y (1999) Structural Aspects of $\mathrm{NaV}_{2} \mathrm{O}_{5}$ under High Pressure. J Phys Soc Jpn 68: 2863291

56. Seyidov MY, Suleymanov RA (2010) Negative Thermal Expansion Due to Negative Area Compressibility in $\mathrm{TlGaSe}_{2}$ Semiconductor with Layered Crystalline Structure. J Appl Phys 108: 06354

57. Cai W, Gładysiak A, Anioła M, Smith VJ, Barbour LJ, Katrusiak A (2015) Giant Negative Area Compressibility Tunable in a Soft Porous Framework Material. J Am Chem Soc 137: 9296-9301

58. Feng G, Zhang W, Dong L, Li W, Cai W, Wei W, Ji L, Lin Z, Lu P (2019) Negative area compressibility of a hydrogen-bonded two-dimensional material. Chem Sci 10: 1309-1315

59. Colmenero F, Sejkora J, Plášil J (2020) Crystal Structure, Infrared Spectrum and Elastic Anomalies in Tuperssuatsiaite. Sci Rep 10: 7510

60. Prawer S, Smith TF, Finlayson TR (1985) The Room Temperature Elastic Behaviour of $\mathrm{CsH}_{2} \mathrm{PO}_{4}$. Aust J Phys 38: 63-83

61. Cairns AB, Catafesta J, Levelut C, Rouquette J, van der Lee A, Peters L, Thompson AL, Dmitriev V, Haines J, Goodwin AL (2013) Giant negative linear compressibility in zinc dicyanoaurate. Nat Mater 12: 212-216

62. Serra-Crespo P, Dikhtiarenko A, Stavitski E, Juan-Alcañiz J, Kapteijn F, Coudert FX, Gascon J (2015) Experimental evidence of negative linear compressibility in the MIL-53 metal-organic framework family. CrystEngComm 17: 276-280

63. Zeng Q, Wang K, Zou B (2017) Large Negative Linear Compressibility in InH(BDC $)_{2}$ from Framework Hinging. J Am Chem Soc 139: 15648-15651

64. Grima JN, Evans KE (2000) Auxetic behavior from rotating squares. J Mater Sci Lett 19: 1563-1565

65. Grima JN, Manicaro E, Attard D (2001) Auxetic behaviour from connected different-sized squares and rectangles, Proc. R. Soc. A 2011, 467, 439-458

66. Grima JN, Zammit V, Gatt R, Alderson A, Evans KE (2007) Auxetic behaviour from rotating semi-rigid units. Phys Status Solidi 244, 866-882

67. Grima-Cornish JN, Vella-Zarb L., Grima JN (2020) Negative Linear Compressibility and Auxeticity in Boron Arsenate. Ann Phys (Berlin) 2020: 1900550

68. Grima JN, Alderson A, Evans KE (2004) Negative Poisson's ratio from rotating rectangles. Comput Methods Sci Technol 10: 137145.

69. Attard D, Grima, JN (2008) Auxetic behaviour from rotating rhombi. Phys Status Solidi (b) 245, 2395-2404

70. Williams JJ, Smith CW, Evans KE, Lethbridge ZDA, Walton RI (2007) An analytical model for producing negative Poisson's ratios and its application in explaining off-axis elastic properties of the NAT-type zeolites. Acta Mater 55, 5697-5706

71. Attard D, Manicaro E, Grima JN. (2009) On rotating rigid parallelograms and their potential for exhibiting auxetic behaviour. Phys Status Solidi (b) 246, 2033-2044.
72. Birkholz M (2006) Thin Film Analysis by X-Ray Scattering. Wiley-VCH Verlag GmbH \& Co., Weinheim, Ch. 1, pp 1-40 